05

\title{
Новый подход к обоснованию формулы Волькенштейна-Птицына для параметра уравнения стеклования
}

\author{
() Д.С. Сандитов, ${ }^{1,2}$ В.В. Мантатов ${ }^{1}$ \\ ${ }^{1}$ Бурятский государственный университет, \\ 670000 Улан-Удэ, Россия \\ ${ }^{2}$ Институт фозического материаловедения СО РАН, \\ 670047 Улан-Удэ, Россия \\ e-mail: sanditov@bsu.ru
}

(Поступило в Редакцию 18 января 2018 г.)

С привлечением концепции Разумовской-Бартенева рассмотрен расширенный вариант интерпретации формулы Волькенштейна-Птицына для полосы температур $\delta T_{g}$, характеризующей интервал перехода от жидкости к стеклу. Предлагаемый авторами вывод этой формулы не зависит от конкретного вида температурной зависимости времени релаксации. У силикатных стекол расчет $\delta T_{g}$ по данной формуле согласуется со способом вычисления с помощью соотношения Вильямса-Ландела-Ферри, а также с левой частью уравнения стеклования $q \tau_{g}=\delta T_{g}$ ( $q$ - скорость охлаждения, $\tau_{g}-$ время релаксации). На базе экспериментальных данных впервые проведен расчет параметров уравнения Разумовской-Бартенева для силикатных стекол и аморфных полимеров.

DOI: $10.21883 / J T F .2018 .12 .46781 .33-18$

\section{Введение}

Переход аморфных веществ из жидкого в твердое стеклообразное состояние носит ярко выраженный релаксационный, кинетический характер. В области перехода молекулярные перегруппировки становятся настолько медленными (время структурной релаксации $\tau$ настолько большим), что изменение структуры не успевает следовать за изменением температуры $T(t)$.

Естественно поэтому, что в процессе стеклования жидкости решающую роль играет соотношение между временем структурной релаксации $\tau$ и скоростью охлаждения расплава $q=(d T / d t)$. В связи с этим приобретает актуальное значение систематическое исследование взаимосвязи этих величин - уравнения стеклования [1-7]:

$$
q \tau_{g}=\delta T_{g}
$$

которое определяет появление стеклообразного состояния при температуре стеклования $T_{g}$ в процессе охлаждения. Здесь $\tau_{g}$ - время структурной релаксации при температуре стеклования. Величина $\delta T_{g}$ имеет смысл полосы температур, характеризующей температурный интервал перехода от жидкости к стеклу [4]. Известно несколько способов оценки $\delta T_{g}$, которые приводят к разным значениям $[1,3-5]$. Причины расхождения результатов расчета $\delta T_{g}$ у разных авторов остаются не совсем ясными.

Эмпирический вариант этого уравнения $\left(q \tau_{q}=C\right)$ предложен Бартеневым [3] в 1951 г. и позже получил обоснование в теории Волькенштейна и Птицына [1]. Была выведена следующая формула для расчета параметра уравнения стеклования $\delta T_{g}[1](\eta-$ вязкость):

$$
\delta T_{g}=-\left(\frac{\partial T}{\partial \ln \tau}\right)_{T=T_{g}} \cong-\left(\frac{\partial T}{\partial \ln \eta}\right)_{T=T_{g}} .
$$

Недавно Немилов [4] показал, что уравнение (1) выводится из теории Мандельштама-Леонтовича [2] и предложил записать эти соотношения в виде равенства (1), вводя обобщенное обозначение $\delta T_{g}$.

Настоящая работа посвящена новому подходу к обоснованию способа расчета величины $\delta T_{g}(2)$, который позволяет вычислять $\delta T_{g}$ непосредственно из экспериментальных данных о вязкости $\eta(T)$ в области стеклования $[4,7]$.

\section{1. Вывод формулы (2) по Волькенштейну-Птицыну}

Авторы [1], исходя из условия стеклования

$$
\left(\frac{d \tau}{d T}\right)_{T=T_{g}}=-\frac{1}{|q|}
$$

и полагая в нем справедливой в первом приближении простую экспоненциальную зависимость $\tau(T)$ при допущении постоянства энергии активации процесса стеклования $U=$ const,

$$
\tau=\tau_{0} \exp \left(\frac{U}{k T}\right),
$$

получили соотношение

$$
q \tau_{g}=\frac{k T_{g}^{2}}{U},
$$


Таблица 1. Параметры уравнений ВЛФ и Разумовской-Бартенева для неорганических стекол и аморфных полимеров

\begin{tabular}{|c|c|c|c|c|c|}
\hline $\begin{array}{l}\text { Аморфное вещество } \\
\text { (состав стекла, mol.\%) }\end{array}$ & $\begin{array}{c}T_{g}, \\
\mathrm{~K}\end{array}$ & $C_{1}$ & $\begin{array}{c}C_{2}, \\
\mathrm{~K}\end{array}$ & $A$ & $B$ \\
\hline $\begin{array}{l}15 \mathrm{Na}_{2} \mathrm{O}-85 \mathrm{SiO}_{2} \\
\mathrm{Na}_{2} \mathrm{O}-80 \mathrm{SiO}_{2} \\
25 \mathrm{Na}_{2} \mathrm{O}-75 \mathrm{SiO}_{2} \\
30 \mathrm{Na}_{2}-70 \mathrm{SiO}_{2} \\
33 \mathrm{Na}_{2} \mathrm{O}-67 \mathrm{SiO}_{2} \\
35 \mathrm{Na}_{2} \mathrm{O}-65 \mathrm{SiO}_{2}\end{array}$ & $\begin{array}{l}782 \\
759 \\
739 \\
721 \\
712 \\
705\end{array}$ & $\begin{array}{l}36 \\
36 \\
35 \\
35 \\
35 \\
35\end{array}$ & $\begin{array}{l}430 \\
390 \\
355 \\
322 \\
304 \\
291\end{array}$ & $\begin{array}{l}65 \\
70 \\
73 \\
78 \\
82 \\
85\end{array}$ & $\begin{array}{l}119 \\
136 \\
152 \\
175 \\
192 \\
205\end{array}$ \\
\hline Оконное стекло & 807 & 36 & 305 & 95 & 252 \\
\hline $\begin{array}{l}69.04 \mathrm{SiO}_{2} \cdot 30.96 \mathrm{Na}_{2} \mathrm{O} \\
43.22 \mathrm{SiO}_{2}-9.55 \mathrm{Na}_{2} \mathrm{O} \cdot 47.23 \mathrm{CsO} \\
79.29 \mathrm{SiO}_{2} \cdot 12.97 \mathrm{Na}_{2} \mathrm{O} \cdot 7.75 \mathrm{Li}_{2} \mathrm{O} \\
71.59 \mathrm{SiO}_{2} \cdot 24.4 \mathrm{Na}_{2} \mathrm{O} \cdot 4.01 \mathrm{Li}_{2} \mathrm{O}\end{array}$ & $\begin{array}{l}718 \\
704 \\
683 \\
681\end{array}$ & $\begin{array}{l}46 \\
31 \\
45 \\
36\end{array}$ & $\begin{array}{l}340 \\
200 \\
315 \\
231\end{array}$ & $\begin{array}{r}97 \\
109 \\
98 \\
106\end{array}$ & $\begin{array}{r}2057 \\
384 \\
211 \\
313\end{array}$ \\
\hline $\begin{array}{l}\text { Оптические стекла } \\
\text { Ф-12 } \\
\text { 3С5 } \\
\text { К2 } \\
\text { БК-9 } \\
\text { ТК-2 }\end{array}$ & $\begin{array}{l}759 \\
829 \\
844 \\
851 \\
887\end{array}$ & $\begin{array}{l}34 \\
32 \\
32 \\
32 \\
31\end{array}$ & $\begin{array}{l}196 \\
196 \\
216 \\
192 \\
199\end{array}$ & $\begin{array}{l}132 \\
135 \\
125 \\
142 \\
138\end{array}$ & $\begin{array}{l}510 \\
572 \\
489 \\
629 \\
616\end{array}$ \\
\hline $\begin{array}{l}\text { Полиизобутилен } \\
\text { Полиуретан } \\
\text { Полиметилакрилат } \\
\text { Поливинилхлорацетат } \\
\text { Натуральный каучук } \\
\text { Поливинилацетат } \\
\text { Метакрилатные полимеры } \\
\text { этиловый } \\
\text { н-бутиловый } \\
\text { н-октиловый }\end{array}$ & $\begin{array}{l}202 \\
238 \\
276 \\
296 \\
300 \\
305 \\
\\
335 \\
300 \\
253\end{array}$ & $\begin{array}{l}38 \\
36 \\
42 \\
40 \\
38 \\
36 \\
\\
40 \\
39 \\
37\end{array}$ & $\begin{array}{r}104 \\
33 \\
45 \\
40 \\
54 \\
47 \\
\\
65 \\
97 \\
107\end{array}$ & $\begin{array}{r}74 \\
260 \\
258 \\
296 \\
211 \\
234 \\
\\
206 \\
121 \\
87\end{array}$ & $\begin{array}{r}143 \\
1872 \\
1580 \\
2190 \\
1173 \\
1516 \\
\\
1062 \\
373 \\
207\end{array}$ \\
\hline
\end{tabular}

а также выражение, которое следует из зависимости (4) при $U=$ const и $T=T_{g}$,

$$
\frac{U}{k T_{g}^{2}}=-\left(\frac{\partial \ln \tau}{\partial T}\right)_{T=T_{g}} .
$$

Из соотношений (1), (5) и (6) следует обсуждаемая формула Волькенштейна-Птицына[1] (2).

С одной стороны, в рассмотренном выводе не учитывается температурная зависимость энергии активации процесса стеклования в области перехода жидкостьстекло. Хорошо известно, что величина $U(T)$ резко возрастает при приближении к температуре стеклования и наблюдается отклонение от экспоненциальной температурной зависимости времени структурной релаксации (4) $[8,9]$. С другой стороны, из конечной формулы Волькенштейна-Птицына (2) следует, что полоса температур $\delta T_{g}$ не зависит от конкретного вида функции $\tau(T)$ и от температурной зависимости энергии активации $U(T)$, поскольку в производной (2) зависимость $\tau(T)$ является непрерывной монотонной функцией $[4,6]$. Из этих соображений следует, что должен существовать более общий подход к выводу данного соотношения.

\section{2. Новый подход к обоснованию формулы (2)}

В настоящее время нет общепризнанной теоретической формулы для зависимости $\tau(T)$ в области стеклования. В связи с этим заслуживает внимания уравнение, полученное Разумовской и Бартеневым [10] без конкретизации вида температурной зависимости $\tau(T)$ :

$$
\begin{gathered}
\ln a_{T}=-\left(\frac{A^{2}}{B}\right) \frac{T-T_{g}}{T-T_{g}\left(1-\frac{A}{B}\right)}, a_{T}=\frac{\tau(T)}{\tau\left(T_{g}\right)} \cong \frac{\eta(T)}{\eta\left(T_{g}\right)}, \\
A=-\left.\frac{\partial \ln \tau}{\partial\left(T / T_{g}\right)}\right|_{T=T_{g}}, \\
B=\left.\frac{1}{2} \frac{\partial^{2} \ln \tau}{\partial\left(T / T_{g}\right)^{2}}\right|_{T=T_{g}} .
\end{gathered}
$$

Параметр $A$ является положительной величиной, так как $\partial \ln \tau>0$ при $\partial\left(T / T_{g}\right)<0$.

Вывод этого соотношения приводится в Приложении. В работе Разумовской и Бартенева [10] дается лишь конечный результат (7) без выкладок. Можно убедиться, 
что математические приближения, использованные при выводе данного уравнения (см. Приложение), универсальны и вполне приемлемы вблизи температуры стеклования.

Подстановка $\tau(T)$ из данной формулы (7) в общее условие стеклования (3) приводит к выражению

$$
q \tau_{g}=\frac{T_{g}}{A}
$$

откуда с учетом производной $A(8)$ приходим к равенству Волькенштейна-Птицына (2)

$$
\delta T_{g}=\frac{T_{g}}{A}=-\left(\frac{\partial T}{\partial \ln \tau}\right)_{T=T_{g}} .
$$

Прежде всего, здесь отметим важный момент, заключающийся в том, что в предлагаемом выводе формула (2) не зависит от вида функциональной зависимости $\tau(T)$. Таким образом, выражение (2) носит более общий характер, чем в интерпретации Волькенштейна и Птицына[1].

\section{3. Соотношение \\ Вильямса-Ландела-Ферри и расчет параметров уравнения Разумовской-Бартенева}

Соотношение (7) совпадает с известным эмпирическим уравнением ВЛФ (Вильямса-ЛанделаФерри) [11,12]

$$
\ln a_{T}=-C_{1} \frac{T-T_{g}}{T-T_{g}+C_{2}},
$$

которое вполне удовлетворительно описывает зависимости $\tau(T)$ и $\eta(T)$ в области стеклования для различных аморфных веществ [5,9,11-16]. Отсюда следует, что эмпирические параметры $C_{1}$ и $C_{2}$ определяются производными $A(8)$ и $B(9)$ :

$$
C_{1}=\frac{A^{2}}{B}, C_{2}=\frac{A}{B} T_{g} .
$$

Зная значения $C_{1}$ и $C_{2}$, с помощью выражений (12) можно рассчитать параметры уравнения РазумовскойБартенева (7). Вычисления проведены для ряда неорганических стекол и аморфных полимеров по формулам (табл. 1)

$$
\begin{gathered}
A=\frac{C_{1}}{C_{2}} T_{g}, \\
B=\frac{A^{2}}{C_{1}}=\frac{C_{1} T_{g}^{2}}{C_{2}^{2}} .
\end{gathered}
$$

В соответствии с соотношениями (1), (10) и (13) полоса температур $\delta T_{g}$ равна отношению параметров ВЛФуравнения (11)

$$
\delta T_{g}=\frac{T_{g}}{A}=\frac{C_{2}}{C_{1}} .
$$

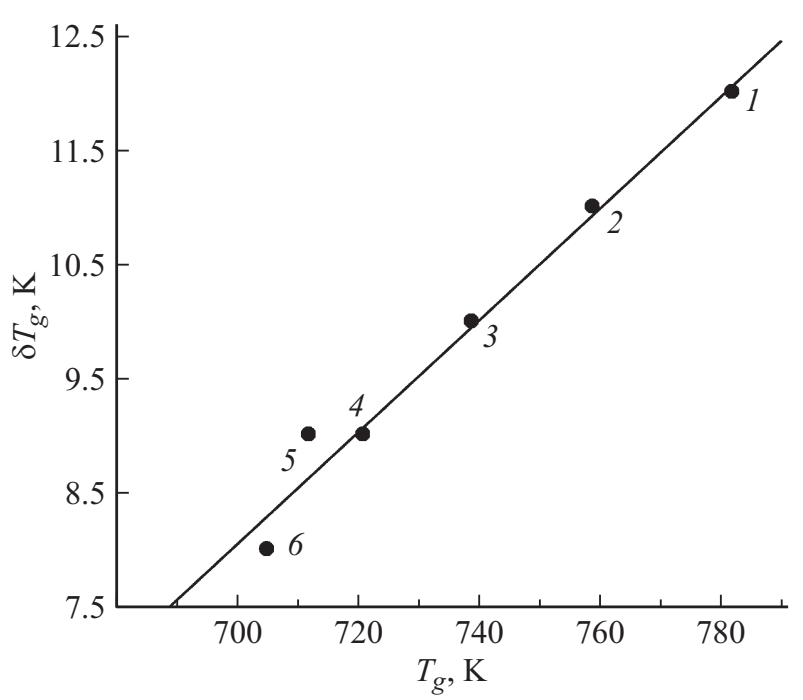

Рис. 1. Линейная корреляция между полосой температур $\delta T_{g}$, характеризующей температурный интервал перехода от жидкости к стеклу, и температурой стеклования $T_{g}$ натриевосиликатных стекол $\mathrm{Na}_{2} \mathrm{O}-\mathrm{SiO}_{2}$. Содержание $\mathrm{Na}_{2} \mathrm{O}, \mathrm{mol} . \%$ : 15 (1), 20 (2), 25 (3), 30 (4), 33 (5) и 35 (6). Использованы данные [18].

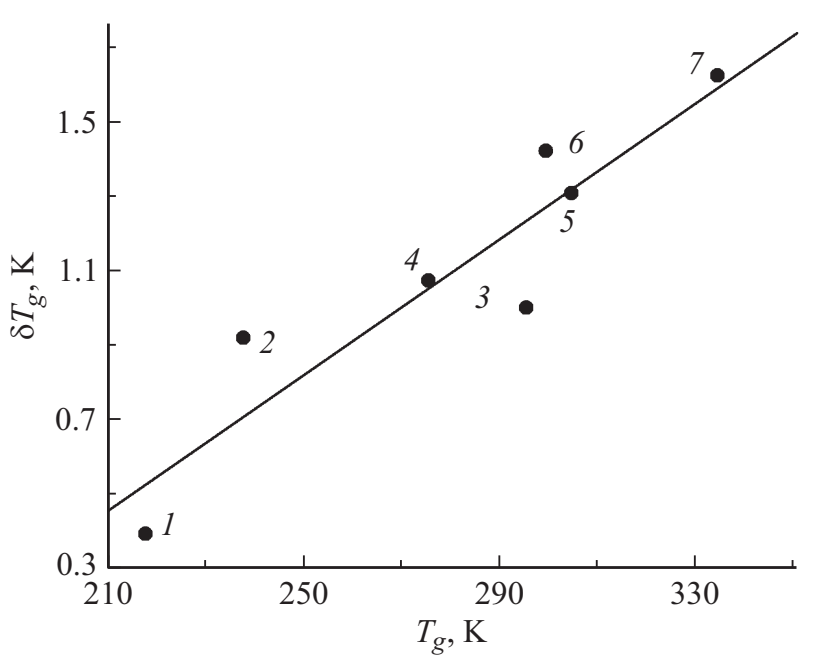

Рис. 2. Корреляция между $\delta T_{g}$ и $T_{g}$ для аморфных органических полимеров: 1 - полигексен-1; 2 - полиуретан; 3 - поливинилхлорацетат; 4 - полиметилакрилат; 5 поливинилацетат; 6 - натуральный каучук; 7 - метакрилат этиловый. Использованы данные [12].

Производная $A(8)$ совпадает с так называемой „фрагильностью“ стеклообразующих расплавов [17]

$$
m=\left.\frac{\partial \ln \eta}{\partial\left(T_{g} / T\right)}\right|_{T=T_{g}},
$$

которая у стекол одного класса практически постоянна: $m \approx$ const. Следовательно, можно ожидать, что и величина $A$ будет постоянной. Поскольку у стекол одного класса величина $A$ слабо зависит от их природы, можно ожидать приближенной линейной корреляции между $\delta T_{g}$ 
Таблица 2. Параметр уравнения стеклования $\delta T_{g}$ для силикатных стекол (использованы данные работы Немилова [4])

\begin{tabular}{|c|c|c|c|}
\hline \multirow{2}{*}{$\begin{array}{l}\text { Силикатные стекла } \\
\text { (состав стекла, mol.\%) }\end{array}$} & \multirow{2}{*}{$\begin{array}{c}T_{g} \\
\mathrm{~K}\end{array}$} & \multicolumn{2}{|c|}{$\delta T_{g}=$} \\
\hline & & $\left(T_{12}-T_{13}\right)$ & $\frac{1}{2.3}\left(T_{12}-T_{13}\right)$ \\
\hline $\begin{array}{l}\text { Оконное }\left(71.2 \mathrm{SiO}_{2} \cdot 1.0 \mathrm{Al}_{2} \mathrm{O}_{3} \cdot 13.9 \mathrm{Na}_{2} \mathrm{O}\right. \\
7.0 \mathrm{CaO} \cdot 6.0 \mathrm{MgO})\end{array}$ & 796 & 19.7 & 8.6 \\
\hline $20 \mathrm{~K}_{2} \mathrm{O} \cdot 80 \mathrm{SiO}_{2}$ & 762 & 20.2 & 8.8 \\
\hline $\begin{array}{l}\mathrm{NBS} 710\left(72.9 \mathrm{SiO}_{2} \cdot 8.9 \mathrm{Na}_{2} \mathrm{O} \cdot 5.1 \mathrm{~K}_{2} \mathrm{O} \cdot 12.9\right. \\
\left.\mathrm{CaO} \cdot 0.2 \mathrm{Sb}_{2} \mathrm{O}_{3}\right)\end{array}$ & 811 & 20.3 & 8.8 \\
\hline $\begin{array}{l}\mathrm{NBS} 711\left(71.7 \mathrm{SiO}_{2} \cdot 3.7 \mathrm{Na}_{2} \mathrm{O} \cdot 5.6 \mathrm{~K}_{2} \mathrm{O} \cdot 19.0\right. \\
\mathrm{PbO})\end{array}$ & 709 & 19.8 & 8.6 \\
\hline $50 \mathrm{PbO} \cdot 50 \mathrm{SiO}_{2}$ & 680 & 14 & 6.1 \\
\hline $\begin{array}{l}\mathrm{C} 92-2\left(74.8 \mathrm{SiO}_{2} \cdot 1.4 \mathrm{Al}_{2} \mathrm{O}_{3} \cdot 5.0 \mathrm{Na}_{2} \mathrm{O} \cdot 7.9\right. \\
\left.\mathrm{K}_{2} \mathrm{O} \cdot 10.9 \mathrm{PbO}\right)\end{array}$ & 706 & 20.2 & 8.8 \\
\hline $45 \mathrm{SiO}_{2} \cdot 26.6 \mathrm{~B}_{2} \mathrm{O}_{3} \cdot 28.4 \mathrm{Na}_{2} \mathrm{O}$ & 773.5 & 12.6 & 5.5 \\
\hline $50.5 \mathrm{SiO}_{2} \cdot 22.0 \mathrm{~B}_{2} \mathrm{O}_{3} \cdot 27.5 \mathrm{Na}_{2} \mathrm{O}$ & 772 & 16.3 & 7.1 \\
\hline $\begin{array}{l}\text { C } 52-1\left(74.4 \mathrm{SiO}_{2} \cdot 19.5 \mathrm{~B}_{2} \mathrm{O}_{3} \cdot 2.3 \mathrm{Al}_{2} \mathrm{O}_{3} \cdot 3.8\right. \\
\left.\mathrm{K}_{2} \mathrm{O}\right)\end{array}$ & 776 & 21.3 & 9.3 \\
\hline $\begin{array}{l}68.9 \mathrm{SiO}_{2} \cdot 2.7 \mathrm{Al}_{2} \mathrm{O}_{3} \cdot 6.7 \mathrm{Na}_{2} \mathrm{O} \cdot 6.6 \mathrm{~K}_{2} \mathrm{O} \cdot 7.4 \\
\mathrm{CaO} \cdot 3.4 \mathrm{MgO} \cdot 0.9 \mathrm{BaO} \cdot 3.4 \mathrm{PbO}\end{array}$ & 732 & 19.4 & 8.4 \\
\hline $\begin{array}{l}72.1 \mathrm{SiO}_{2} \cdot 2.7 \mathrm{Al}_{2} \mathrm{O}_{3} \cdot 8.8 \mathrm{Na}_{2} \mathrm{O} \cdot 5.7 \mathrm{~K}_{2} \mathrm{O} \cdot 2.4 \\
\mathrm{ZnO}\end{array}$ & 759 & 19.5 & 8.5 \\
\hline $\begin{array}{l}67.2 \mathrm{SiO}_{2} \cdot 20 \mathrm{~B}_{2} \mathrm{O}_{3} \cdot 1.3 \mathrm{Al}_{2} \mathrm{O}_{3} \cdot 9.1 \mathrm{~K}_{2} \mathrm{O} \cdot 2.4 \\
\mathrm{ZnO}\end{array}$ & 795 & 22.5 & 9.8 \\
\hline $\begin{array}{l}\text { Оконное }\left(71.8 \mathrm{SiO}_{2} \cdot 0.8 \mathrm{Al}_{2} \mathrm{O}_{3} \cdot 13.0 \mathrm{Na}_{2} \mathrm{O}\right. \\
\left.0.3 \mathrm{~K}_{2} \mathrm{O}_{3} \cdot 9.1 \mathrm{CaO} \cdot 5.0 \mathrm{MgO}\right)\end{array}$ & 779 & 20 & 8.7 \\
\hline
\end{tabular}

Таблица 3. Расчет полосы температур $\delta T_{g}$ для силикатных стекол двумя способами

\begin{tabular}{|c|c|c|c|c|c|c|c|}
\hline Стекло (состав стекла, mol.\%) & $\begin{array}{c}T_{12} \\
\mathrm{~K}\end{array}$ & $\begin{array}{c}T_{13} \\
\mathrm{~K}\end{array}$ & $C_{1}$ & $\begin{array}{c}C_{2} \\
\mathrm{~K}\end{array}$ & $\begin{array}{c}\tau_{g} \\
\mathrm{~s}\end{array}$ & $\begin{array}{c}\delta T_{g}, \\
\mathrm{~K} \\
(18)\end{array}$ & $\begin{array}{c}\delta T_{g}=\frac{C_{2}}{C_{1}}, \\
\mathrm{~K} \\
(19)\end{array}$ \\
\hline \multicolumn{8}{|c|}{ Натриево-силикатные стекла $\mathrm{Na}_{2} \mathrm{O}-\mathrm{SiO}_{2}$ [18] } \\
\hline $\begin{array}{l}15 \mathrm{Na}_{2} \mathrm{O}-85 \mathrm{SiO}_{2} \\
20 \mathrm{Na}_{2} \mathrm{O}-80 \mathrm{SiO}_{2} \\
25 \mathrm{Na}_{2} \mathrm{O}-75 \mathrm{SiO}_{2} \\
30 \mathrm{Na}_{2} \mathrm{O}-70 \mathrm{SiO}_{2} \\
33 \mathrm{Na}_{2} \mathrm{O}-67 \mathrm{SiO}_{2} \\
35 \mathrm{Na}_{2} \mathrm{O}-65 \mathrm{SiO}_{2}\end{array}$ & $\begin{array}{l}819 \\
792 \\
769 \\
749 \\
738 \\
726\end{array}$ & $\begin{array}{l}790 \\
766 \\
745 \\
727 \\
717 \\
705\end{array}$ & $\begin{array}{l}36 \\
36 \\
35 \\
35 \\
35 \\
35\end{array}$ & $\begin{array}{l}430 \\
390 \\
355 \\
322 \\
304 \\
291\end{array}$ & $\begin{array}{l}239 \\
217 \\
202 \\
184 \\
174 \\
166\end{array}$ & $\begin{array}{c}13 \\
11 \\
10 \\
10 \\
9 \\
9\end{array}$ & $\begin{array}{c}12 \\
11 \\
10 \\
9 \\
9 \\
8\end{array}$ \\
\hline Оконное стекло [19] & 846 & 825 & 36 & 305 & 160 & 9 & 8 \\
\hline \multicolumn{8}{|c|}{ Полищелочные силикатные стекла [20] } \\
\hline $\begin{array}{l}\text { 69.04 } \mathrm{SiO}_{2} \cdot 30.96 \mathrm{Na}_{2} \mathrm{O} \\
\text { 79.29 } \mathrm{SiO}_{2} \cdot 12.97 \mathrm{Na}_{2} \mathrm{O} \cdot 7.75 \mathrm{Li}_{2} \mathrm{O} \\
43.22 \mathrm{SiO}_{2} \cdot 9.55 \mathrm{Na}_{2} \mathrm{O} \cdot 47.23 \mathrm{CsO} \\
71.59 \mathrm{SiO}_{2} \cdot 24.4 \mathrm{Na}_{2} \mathrm{O} \cdot 4.01 \mathrm{Li}_{2} \mathrm{O}\end{array}$ & $\begin{array}{l}736 \\
700 \\
721 \\
695\end{array}$ & $\begin{array}{l}718 \\
683 \\
704 \\
681\end{array}$ & $\begin{array}{l}46 \\
45 \\
31 \\
36\end{array}$ & $\begin{array}{l}340 \\
315 \\
200 \\
231\end{array}$ & $\begin{array}{l}147 \\
140 \\
129 \\
128\end{array}$ & $\begin{array}{l}8 \\
7 \\
7 \\
6\end{array}$ & $\begin{array}{l}7 \\
7 \\
6 \\
6\end{array}$ \\
\hline
\end{tabular}

Примечание. Состав оконного стекла (wt.\%) [19]: $\mathrm{SiO}_{2}-72.7 ; \mathrm{CaO}-8.6 ; \mathrm{MgO}-3.4 ; \mathrm{Al}_{2} \mathrm{O}_{3}-1.3 ; \mathrm{Na}_{2} \mathrm{O}-13.6 ; \mathrm{K}_{2} \mathrm{O}-0.4 ; \tau_{g}=C_{2} / q C_{1}$, $C_{1}$ и $C_{2}$ - параметры уравнения Вильямса-Ландела-Ферри, $q=0.05 \mathrm{~K} / \mathrm{s}$.

и температурой стеклования $T_{g}$, что подтверждается для некоторых стеклообразных систем (рис. 1,2).

\section{4. Расчет полосы температур $\delta T_{g}$ для силикатных стекол}

С привлечением методики Немилова [4] на основе экспериментальных данных о температурах $T_{12}$ и
$T_{13}$ (табл. 2,3), соответствующих логарифмам вязкости $\lg \eta_{12}=12$ и $\lg \eta_{13}=13$, рассчитаем $\delta T_{g}$ по формуле Волькенштейна-Птицына (2):

$$
\begin{aligned}
\delta T_{g} & =-\frac{1}{2.3}\left(\frac{\Delta T}{\Delta \lg \eta}\right)_{T_{g}}=-\frac{1}{2.3}\left(\frac{T_{12}-T_{13}}{\lg \eta_{12}-\lg \eta_{13}}\right) \\
& =\frac{1}{2.3}\left(T_{12}-T_{13}\right) .
\end{aligned}
$$


Как и следовало ожидать, полученный результат для ряда силикатных стекол (табл. 3)

$$
\delta T_{g}=\frac{1}{2.3}\left(T_{12}-T_{13}\right)=(6-13) \mathrm{K}
$$

хорошо согласуется с данными для этих же стекол, рассчитанными по соотношению (15) с помощью уравнения ВЛФ (табл. 3):

$$
\delta T_{g}=\frac{C_{2}}{C_{1}}=(6-12) \mathrm{K},
$$

а также с произведением $q \tau_{g}-\mathrm{c}$ левой частью уравнения стеклования (1) [5]

$$
q \tau_{g} \approx(5-12) \mathrm{K} .
$$

В отличие от подхода Немилова [4] мы не вводим в формулу (2) эмпирический множитель 2.3. Поэтому наш результат (18) примерно в 2 раза меньше, чем оценка Немилова [4] (табл. 2)

$$
\delta T_{g}=\left(T_{12}-T_{13}\right) \approx 20 \mathrm{~K} .
$$

По приближенной оценке Волькенштейна-Птицына [1] величина $\delta T_{g}$ составляет несколько градусов. Одним из авторов настоящей работы получены значения $\delta T_{g} \approx(5-10) \mathrm{K}$ для неорганических стекол и $\delta T_{g} \approx(1-3) \mathrm{K}$ для органических полимерных стекол [5]. Бартеневым [21] были предложены более высокие значения: $\delta T_{g} \approx 20 \mathrm{~K}$ у силикатных стекол и $\delta T_{g} \approx 10 \mathrm{~K}$ у аморфных полимеров. По классическим представлениям Саймона (Simon) [22] структура стеклообразующей жидкости должна замораживаться в очень узкой области температур, включающей $T_{g}$.

В дальнейшем важно продолжить поиски корректных способов расчета параметра $\delta T_{g}$, характеризующего температурный интервал перехода жидкости в стекло [1-7].

\section{Заключение}

Предложен новый подход к интерпретации формулы Волькенштейна-Птицына для параметра уравнения стеклования $\delta T_{g}$. Предлагаемый вывод этой формулы не зависит от конкретного вида зависимости $\tau(T)$ и от зависимости энергии активации процесса стеклования от температуры $U(T)$. Показано, что способ нахождения $\delta T_{g}$ по Волькенштейну-Птицыну $\left(\delta T_{g} \approx(6-12) \mathrm{K}\right)$ и метод оценки $\delta T_{g}$ с привлечением уравнения ВЛФ $\left(\delta T_{g} \approx(8-12) \mathrm{K}\right)$ у силикатных стекол находятся в согласии с произведением $q \tau_{g} \approx(5-12) \mathrm{K}$. Проведен расчет параметров уравнения Разумовской-Бартенева для неорганических стекол и аморфных органических полимеров.

Авторы благодарят проф. И.В. Разумовскую за консультацию по выводу уравнения Разумовской-Бартенева и обсуждение результатов.

Работа выполнена при финансовой поддержке Минобрнауки РФ (грант № 3.5406.2017/8.9).

\section{Приложение. Вывод уравнения (7)}

Рассмотрим разложение в ряд функции $\ln \eta(T)$ (или $\ln \tau(T))$ вблизи $T_{g}$ по малому безразмерному параметру $\lambda$ - относительному приращению температуры вблизи $T_{g}$

$$
\lambda=\frac{T-T_{g}}{T_{g}} .
$$

Полагаем, что применительно к нашей задаче вполне достаточно ограничиться первыми тремя членами разложения

$$
\begin{aligned}
\ln \eta(T) & \cong \ln \eta\left(T_{g}\right)-A \lambda+B \lambda^{2}, \\
A & =-\left.\frac{\partial \ln \eta}{\partial\left(T / T_{g}\right)}\right|_{T=T_{g}}, \\
B & =\left.\frac{1}{2} \frac{\partial^{2} \ln \eta}{\partial\left(T / T_{g}\right)^{2}}\right|_{T=T_{g}} .
\end{aligned}
$$

Вводя относительную вязкость $a_{T}=\eta(T) / \eta\left(T_{g}\right)$

$$
\ln a_{T}=\ln \eta(T)-\ln \eta\left(T_{g}\right),
$$

представим выражение (П2) в виде

$$
\ln a_{T}=-A \lambda+B \lambda^{2}=-A \lambda\left(1-\frac{B \lambda}{A}\right) .
$$

Рассмотрим это уравнение (П5) при таких значениях $\lambda$, когда можно считать, что в разложении (П2) третий член значительно меньше второго члена: $B \lambda^{2} \ll A \lambda$, откуда следует неравенство

$$
x=\frac{B \lambda}{A} \ll 1,
$$

которое, как правило, выполняется вблизи $T_{g}$ (из-за малости $\lambda$ ). Поэтому можем воспользоваться известным приближением

$$
1+x \cong \frac{1}{1-x},
$$

где $x=B \lambda / A$ с учетом $\lambda(T)$ (П1) является функцией температуры

$$
x=\frac{B\left(T-T_{g}\right)}{A T_{g}} .
$$

Далее, подставив (П8) в равенство (П7), а затем (П7) в выражение (П5), приходим к формуле (7) [10,23]:

$$
\ln a_{T}=-\left(\frac{A^{2}}{B}\right) \frac{T-T_{g}}{T-T_{g}\left(1-\frac{A}{B}\right)},
$$

где $A$ и $B$ определены производными (П3) и (П4).

Таким образом, универсальность уравнения ВЛФ (11) вытекает из разложения функции $\ln \eta(T)$ в ряд, аналогично универсальности закона Гука (гармоническое приближение). 


\section{Список литературы}

[1] Волькенштейн М.В., Птицын О.Б. // ЖТФ. 1956. Т. 26. Вып. 10. С. 2204-2222.

[2] Мандельштам Л.И., Леонтович М.А. // ЖЭТФ. 1937. Т. 7. № 3. С. 438-449.

[3] Бартенев Г.М. // ДАН СССР. 1951. Т. 76. № 2. С. 227-230.

[4] Немилов С.В. // ФХС. 2013. Т. 39. № 6. С. 857-878.

[5] Сандитов Д.С. // ЖЭТФ. 2016. Т. 150. Вып. 3 (9). C. $501-515$.

[6] Немилов С.В. // ЖЭТФ. 2017. Т. 151. Вып. 5. С. 891-892.

[7] Сандитов Д.С. // ЖЭТФ. 2017. Т. 151. Вып. 5. С. 893-896.

[8] Ростиашвили В.Г., Иржак В.И., Розенберг Б.А. Стеклование полимеров. Л.: Химия, 1987. 192 с.

[9] Сандитов Д.С., Бартенев Г.М. Физические свойства неупорядоченных структур. Новосибирск: Наука, 1982. 259 с.

[10] Разумовская И.В., Бартенев Г.М. Стеклообразное состояние. Тр. V Всес. совещ. Л.: Наука, 1971. С. 34.

[11] Williams M.L., Landel R.F., Ferry J.D. // J. Am. Chem. Soc. 1955. Vol. 77. N 14. P. 3701-3707.

[12] Ferry J.D. Viscoelastic Properties of Polymers. NY.: Marcel Dekker, 1970. 671 р. [Ферри Джк. Вязкоупругие свойства полимеров. М.: ИЛ, 1963. 535 с.]

[13] Bestul B.A. // Glastechn. Ber. 1959. Bd. K32. S. 59-64.

[14] Сандитов Д.С., Доржиев Д.Б., Балданов Ж.П. // ЖФХ. 1973. Т. 47. № 12. С. 2990-2994.

[15] Бадмаев С.С., Сангадиев С.Ш., Сандитов Д.С. // Вестник Бурятского гос. ун-та. Физика. Химия. 2015. Вып. 3. C. $99-104$.

[16] Дуров В.А., Шахпаронов М.И. // ЖФХ. 1979. Т. 53. № 10. C. $2456-2459$.

[17] Angell C.A. // J. Phys. Chem. Sol. 1988. Vol. 49. N 8. P. 836-871.

[18] MDL \& SciGlass-7.8. Institute of Theoretical Chemistry, Shrewsbury, MA, 2012.

[19] Бартенев Г.М., Сандитов Д.С. Релаксационные процессы в стеклообразных системах. Новосибирск: Наука, 1986. $235 \mathrm{c}$.

[20] Столяр С.В., Беседина С.А. // ФХС. 1992. Т. 18. № 3. C. $88-95$.

[21] Бартенев Г.М. Строение и механические свойства неорганических стекол. М.: Стройиздат, 1966. 216 с.

[22] Simon F. // Z. Anorg. Allg. Chem. 1931. Bd. 203. N 1-2. S. $219-227$.

[23] Сандитов Д.С., Разумовская И.В. // Высокомолек. соед. Cep. A. 2018. T. 60. № 2. С. 110-115. 